\title{
Study of the turbulence models over an aircraft wing
}

\section{Étude des Modèles de Turbulence sur une Aile d'Avion}

\author{
Rabii El Maani ${ }^{1}$, Soufiane Elouardi ${ }^{2}$, Bouchaib Radi ${ }^{3}$, Abdelkhalek El Hami ${ }^{4}$ \\ ${ }^{1}$ LSMI, ENSAM Meknès, Marjane 2, Maroc, elmaani.rabi3@gmail.com \\ ${ }^{2}$ LIMII, FST Settat, Route de Casablanca, Maroc, so.elouardi@uhp.ac.ma \\ ${ }^{3}$ LIMII, FST Settat, Route de Casablanca, Maroc, bouchaib.radi@yahoo.fr \\ ${ }^{4}$ LMN, INSA de Rouen, France, abdelkhalak.elhami@insa-rouen.fr
}

RÉSUMÉ. L'aérodynamique est définie comme la science de la manipulation d'un fluide qui est souvent l'air en interaction avec une structure. Lors de la simulation de l'écoulement au-dessus des pales aérodynamiques, la transition d'un écoulement laminaire à un écoulement turbulent joue un rôle important dans la détermination des caractéristiques d'écoulement et dans la quantification des performances de la surface portante telles que la portance et la traînée. Ces flux fluidiques sont soumis à des contraintes visqueuses et à une inertie qui produit des fluctuations désordonnées. La turbulence affecte donc le comportement du flux aérodynamique ainsi que la structure en interaction avec un fluide pour des nombres de Reynolds élevés. En effet, il est nécessaire de contrôler ces flux turbulents dans ce domaine afin de donner une bonne conception de la structure. Plusieurs modèles de turbulence ont été développés pour faciliter le calcul des grandeurs caractéristiques afin d'optimiser la simulation des écoulements turbulents en aérodynamique. Dans cet article, nous avons présenté une validation d'une simulation numérique d'un écoulement transsonique 3D sur l'aile ONERA M6 pour laquelle les résultats numériques, réalisés avec ANSYS/FLUENT @ , seront comparés à des données expérimentales et à des résultats numériques de la NASA portant sur le coefficient de pression $(\mathrm{Cp})$ le long des surfaces des ailes supérieures et inférieures. Le flux a été obtenu en résolvant les équations de conservation de la masse et de la quantité de mouvement en régime permanent, combinées à l'un des cinq modèles de turbulence (Spalart-Allmaras (S-A), k- $\varepsilon$ standard, $k-\varepsilon$ RNG, $k-\omega$ standard et $k-\omega$ SST) visant à la validation de ces modèles par la comparaison des prévisions et des mesures expérimentales en champ libre pour l'aile sélectionnée. ABSTRACT. Aerodynamics is defined as the science of handling a fluid that is often the air interacting with a structure. When simulating the flow over airfoils, transition from laminar to turbulent flow plays an important role in determining the flow features and in quantifying the airfoil performance such as lift and drag. These fluidic flows are subjected to viscous stresses and inertia which produces disordered fluctuations, so turbulence affects the behavior of the aerodynamic flow as well as the structure interacting with the fluid in a range of high Reynolds, indeed, it is obliged to control these turbulent flows in this area in order to give a good design of the structure. Several models of turbulence have been developed to facilitate the calculation of characteristic quantities to optimize the simulation of turbulent flows in aerodynamics. In this paper, we carried out a validation of a numerical simulation of a 3D transonic flow over the ONERA M6 wing for which the numerical results, performed using ANSYS/FLUENTC, will be compared with experimental data and NASA CFD results consisting on the pressure coefficient $(\mathrm{Cp})$ along the upper and lower wing surfaces. The flow was obtained by solving the steady-state governing equations of continuity and momentum conservation combined with one of five turbulence models (Spalart-Allmaras (S-A), standard k- $\varepsilon$, k- $\varepsilon$ RNG, standard k- $\omega$ and k- $\omega$ SST) aiming to the validation of these models through the comparison of the predictions and the free field experimental measurements for the selected wing.

MOTS-CLÉS. Aérodynamique, CFD, aile ONERA M6, modèles de turbulence.

KEYWORDS. Aerodynamics, CFD, ONERA M6 wing, turbulence models.

\section{Introduction}

In the aeronautical industry, in recent years the aerodynamic and structural optimization of aircraft wings has been the subject of much research [ELM 16]. Aerodynamics is defined as the science of handling a fluid that is often the air interacting with a structure. In this science, the number of research is increasing rapidly due to the rapid evolution of computational fluid dynamics (CFD) [ELM 17]. These fluidic flows are subjected to viscous stresses and inertia which produces disordered fluctuations, so turbulence affects the behavior of the aerodynamic flow as well as the structure interacting with the fluid in a range of high Reynolds. Numerical simulation was motivated by the need for faster and more accurate methods for calculating flow fields around a technical configuration.

When simulating flow on aerodynamic profiles, turbulent fluctuations play an important role in the identification and quantification of aerodynamic flow characteristics. On the one hand, aerodynamic tests provide accurate results, but they are very complex and expensive. On the other hand, there are (c) 2018 ISTE OpenScience - Published by ISTE Ltd. London, UK - openscience.fr 
analytical and semi-empirical models, which adopt simplifying assumptions, but they are not universally reliable. Computational Fluid Dynamics (CFD) offers the best or alternative support for direct measurement, and computer simulations show features and details that are difficult, expensive or impossible to measure or visualize experimentally. In principle, both laminar and turbulent flows are described using the Navier-Stokes equations without need of additional information. To help predict the effects of turbulence, a large amount of Computational Fluid Dynamics (CFD) research has focused on methods using turbulence models [WIL 94]. These turbulence models have been specially developed to account for turbulence effects.

This paper aims to analyze the aerodynamic interactions of a three-dimensional ONERA-M6 wing at transonic speeds and very high Reynolds numbers in order to validate numerical simulations of flows. ONERA-M6 wing results database was used for the comparison and evaluation of five turbulence models such as Spalart-Allmaras model, k- $\varepsilon$ Standard, k- $\varepsilon$ RNG, k- $\omega$ Standard and k- $\omega$ SST, in order to show the fluid flow behavior at these conditions and to establish a verified solution method.

\section{Mathematical formulation and turbulence models}

In general, turbulence models attempt to modify the original unsteady Navier-Stokes equations by introducing averaged and fluctuating quantities to produce Reynolds Averaged Navier-Stokes equations (RANS). These equations represent only the main quantities of the flux, while modeling the effects of turbulence without the need to solve turbulent fluctuations. All scales of the turbulence field are modeled. The turbulence models based on the RANS equations are known as statistical turbulence models because of the statistical mean procedure used to obtain the equations. The starting point of any numerical flow simulation is the set of Navier-Stokes equations in their instantaneous form plus the fluid state equation for closing the system.

Two alternative methods can be employed to render the Navier-Stokes equations tractable so that the small-scale turbulent fluctuations do not have to be directly simulated: Reynolds-averaging (or ensemble-averaging) and filtering. Both methods introduce additional terms in the governing equations that need to be modeled in order to achieve a "closure" for the unknowns.

The Reynolds mean Navier-Stokes (RANS) equations for an unsteady flow of a compressible fluid can be written on the spatial domain as:

$$
\begin{aligned}
& \frac{\partial \rho}{\partial t}+\frac{\partial}{\partial x_{i}}\left(\rho u_{i}\right)=0 \\
& \frac{\partial}{\partial t}\left(\rho u_{i}\right)+\frac{\partial}{\partial x_{i}}\left(\rho u_{i} u_{j}\right)=-\frac{\partial p}{\partial x_{i}}+\frac{\partial}{\partial x_{j}}\left[\mu\left(\frac{\partial u_{i}}{\partial x_{j}}+\frac{\partial u_{j}}{\partial x_{i}}-\frac{2}{2} \delta_{i j} \frac{\partial u_{l}}{\partial x_{l}}\right)\right]+\frac{\partial}{\partial x_{j}}\left(-\rho \overline{u_{i}^{\prime} u_{j}^{\prime}}\right)
\end{aligned}
$$

\subsection{Spalart-Allmaras (S-A) model}

The Spalart-Allmaras model is a relatively simple one-equation model that solves a modeled transport equation for the kinematic eddy (turbulent) viscosity [SPA 93]. This embodies a relatively new class of one-equation models in which it is not necessary to calculate a length scale related to the local shear layer thickness. The Spalart-Allmaras model was designed specifically for aerospace applications involving wall-bounded flows and has been shown to give good results for boundary layers subjected to adverse pressure gradients. It is also gaining popularity for turbomachinery applications.

The transported variable in the Spalart-Allmaras model $\tilde{v}$ is identical to the turbulent kinematic viscosity except in the near-wall (viscous-affected) region. The transport equation for $\tilde{v}$ is: 


$$
\frac{\partial}{\partial t}(\rho \tilde{v})+\frac{\partial}{\partial x_{i}}\left(\rho \tilde{v} u_{i}\right)=G_{v}+\frac{1}{\sigma_{\tilde{v}}}\left[\frac{\partial}{\partial x_{i}}\left\{(\mu+\rho \tilde{v}) \frac{\partial \tilde{v}}{\partial x_{i}}\right\}+C_{b 2} \rho\left(\frac{\partial \tilde{v}}{\partial x_{i}}\right)^{2}\right]-Y_{v}+S_{\tilde{v}}
$$

Where $G_{v}$ is the production of turbulent viscosity, $Y_{v}$ is the destruction of turbulent viscosity that occurs in the near-wall region due to wall blocking and viscous damping, $\sigma_{\tilde{v}}$ and $C_{b 2}$ are constants, $v$ is the molecular kinematic viscosity and $S_{\tilde{v}}$ is a user-defined source term. Note that since the turbulence kinetic energy $k$ is not calculated in the Spalart-Allmaras model.

The production term $G_{v}$ is modeled as:

$$
G_{v}=C_{b 1} \rho \tilde{S} \tilde{v}
$$

Where: $\tilde{S} \equiv S+\frac{\tilde{v}}{\kappa^{2} d^{2}} f_{v 2}$ and $f_{v 2}=1-\frac{\chi}{1+\chi f_{v 1}}$

$C_{b 1}$ and $\kappa$ are constants, $\mathrm{d}$ is the distance from the wall and $S$ is a scalar measure of the deformation tensor.

The destruction term is modeled as:

$$
Y_{v}=C_{\omega 1} \rho f_{\omega}\left(\frac{\tilde{v}}{d}\right)^{2}
$$

Where: $f_{\omega}=g\left[\frac{1+C_{\omega 3}^{6}}{g^{6}+C_{\omega 3}^{6}}\right]^{1 / 6}, g=r+C_{\omega 2}\left(r^{6}-r\right)$ and $r \equiv \frac{\tilde{v}}{\tilde{S} \kappa^{2} d^{2}}$

$C_{\omega 1}, C_{\omega 2}$ and $C_{\omega 3}$ are constants.

The model constants have following values:

$$
\begin{aligned}
& C_{b 1}=0.1355, C_{b 2}=0.622, \sigma_{\tilde{\nu}}=\frac{2}{3}, C_{v 1}=7.1, \\
& C_{\omega 1}=3.239, C_{\omega 2}=0.3, C_{\omega 3}=2.0, \kappa=0.4187
\end{aligned}
$$

\section{2. $k-\varepsilon$ standard model}

The simplest "complete models" of turbulence are two-equation models in which the solution of two separate transport equations allows the turbulent velocity and length scales to be independently determined. Two-equation turbulence models allow the determination of both, a turbulent length and time scale by solving two separate transport equations.

The standard $k-\varepsilon$ model [LAU 72] is a model based on model transport equations for the turbulence kinetic energy $(k)$ and its dissipation rate $(\varepsilon)$. The model transport equation for $k$ is derived from the exact equation, while the model transport equation for $\varepsilon$ was obtained using physical reasoning and bears little resemblance to its mathematically exact counterpart.

In the derivation of the $k-\varepsilon$ model, the assumption is that the flow is fully turbulent, and the effects of molecular viscosity are negligible. The standard $k-\varepsilon$ model is therefore valid only for fully turbulent flows. 
The turbulence kinetic energy $k$ and its rate of dissipation $\varepsilon$ are obtained from the following transport equations:

$$
\begin{aligned}
& \frac{\partial}{\partial t}(\rho k)+\frac{\partial}{\partial x_{i}}\left(\rho k u_{i}\right)=\frac{\partial}{\partial x_{j}}\left[\left(\mu+\frac{\mu_{t}}{\sigma_{k}}\right) \frac{\partial k}{\partial x j}\right]+G_{k}+G_{b}-\rho \varepsilon-Y_{M}+S_{k} \\
& \frac{\partial}{\partial t}(\rho \varepsilon)+\frac{\partial}{\partial x_{i}}\left(\rho \varepsilon u_{i}\right)=\frac{\partial}{\partial x_{j}}\left[\left(\mu+\frac{\mu_{t}}{\sigma_{\varepsilon}}\right) \frac{\partial \varepsilon}{\partial x j}\right]+C_{1 \varepsilon} \frac{\varepsilon}{k}\left(G_{k}+C_{3 \varepsilon} G_{b}\right)-C_{2 \varepsilon} \rho \frac{\varepsilon^{2}}{k}+S_{\varepsilon}
\end{aligned}
$$

$G_{k}$ represents the generation of turbulence kinetic energy due to the mean velocity gradients, $G_{b}$ is the generation of turbulence kinetic energy due to buoyancy, $Y_{M}$ represents the contribution of the fluctuating dilatation in compressible turbulence to the overall dissipation rate, $C_{1 \varepsilon}, C_{2 \varepsilon}$ and $C_{3 \varepsilon}$ are constants, $\sigma_{k}$ and $\sigma_{\varepsilon}$ are the turbulent Prandtl numbers for $k$ and $\varepsilon$, respectively, $S_{k}$ and $S_{\varepsilon}$ are source terms.

The turbulent (or eddy) viscosity $\mu_{t}$ is computed by combining $k$ and $\varepsilon$ as follows:

$$
\mu_{t}=\rho C_{\mu} \frac{k^{2}}{\varepsilon}
$$

Where $C_{\mu}$ is a constant.

The model constants have following values:

$$
C_{1 \varepsilon}=1.44, \quad C_{2 \varepsilon}=1.92, \quad C_{\mu}=0.09, \quad \sigma_{k}=1.0, \sigma_{\varepsilon}=1.3
$$

\section{3. $k-\varepsilon R N G$ model}

The $k-\varepsilon$ RNG turbulence model is derived from the instantaneous Navier-Stokes equations, using a mathematical technique called renormalization group RNG methods.

The $k-\varepsilon$ RNG model has a similar form to the standard $k-\varepsilon$ model:

$$
\begin{aligned}
& \frac{\partial}{\partial t}(\rho k)+\frac{\partial}{\partial x_{i}}\left(\rho k u_{i}\right)=\frac{\partial}{\partial x_{j}}\left(\alpha_{k} \mu_{e f f} \frac{\partial k}{\partial x_{j}}\right)+G_{k}+G_{b}-\rho \varepsilon-Y_{M}+S_{k} \\
& \frac{\partial}{\partial t}(\rho \varepsilon)+\frac{\partial}{\partial x_{i}}\left(\rho \varepsilon u_{i}\right)=\frac{\partial}{\partial x_{j}}\left(\alpha_{\varepsilon} \mu_{e f f} \frac{\partial \varepsilon}{\partial x j}\right)+C_{1 \varepsilon} \frac{\varepsilon}{k}\left(G_{k}+C_{3 \varepsilon} G_{b}\right)-C_{2 \varepsilon} \rho \frac{\varepsilon^{2}}{k}-R_{\varepsilon}+S_{\varepsilon}
\end{aligned}
$$

$G_{k}$ represents the generation of turbulence kinetic energy due to the mean velocity gradients, $G_{b}$ is the generation of turbulence kinetic energy due to buoyancy, $Y_{M}$ represents the contribution of the fluctuating dilatation in compressible turbulence to the overall dissipation rate, $\sigma_{k}$ and $\sigma_{\varepsilon}$ are the inverse effective Prandtl numbers for $k$ and $\varepsilon$, respectively, $S_{k}$ and $S_{\varepsilon}$ are source terms.

The scale elimination procedure in RNG theory results in a differential equation for turbulent viscosity:

$$
d\left(\frac{\rho^{2} k}{\sqrt{\varepsilon \mu}}\right)=1.72 \frac{\hat{v}}{\sqrt{\hat{v}^{3}-1+C_{v}}} d \hat{v}
$$


Where $\hat{v}=\frac{\mu_{e f f}}{\mu}$ and $C_{v} \approx 100$

The main difference between the RNG and standard $k-\varepsilon$ models lies in the additional term in the $\varepsilon$ equation given by:

$$
R_{\varepsilon}=\frac{C_{\mu} \rho \eta^{3}\left(1-\frac{\eta}{\eta_{0}}\right)}{1+\beta \eta^{3}} \frac{\varepsilon^{2}}{k}
$$

Where: $\eta \equiv \frac{S k}{\omega}, \eta_{0}=4.38, \beta=0.012, C_{1 \varepsilon}=1.42$ and $C_{2 \varepsilon}=1.68$

The production of turbulence kinetic energy $G_{k}$ is modeled identically for the standard $k-\varepsilon$ model:

$$
G_{k}=-\rho \overline{u_{i}^{\prime} u_{j}^{\prime}} \frac{\partial u_{j}}{\partial x_{i}}
$$

To evaluate $G_{k}$ in a manner consistent with the boussinesq hypothesis:

$$
G_{k}=\mu_{t} S^{2}
$$

Where $S$ is the modulus of the mean rate of strain tensor, defined as:

$$
S \equiv \sqrt{2 S_{i j} S_{i j}}
$$

When using the high Reynolds number $k-\varepsilon$ versions, $\mu_{e f f}$ is used instead of $\mu_{t}$.

\section{4. $k$ - $\omega$ standard model}

The standard $k-\omega$ model is based on the Wilcox $k-\omega$ model [WIL 94] which incorporates modifications for low-Reynolds number effects, compressibility and shear flow spreading. One of the weak points of the Wilcox model is the sensitivity of the solutions to values for $k$ and $\omega$ outside the shear layer (freestream sensitivity).

The standard $k-\omega$ model is an empirical model based on model transport equations for the turbulence kinetic energy $(k)$ and the specific dissipation rate $(\omega)$, which can also be thought of as the ratio of $k$ to $\varepsilon$.

As the $k-\omega$ model has been modified over the years, production terms have been added to both the $k$ and $\omega$ equations, which have improved the accuracy of the model for predicting free shear flows.

The turbulence kinetic energy, $k$ and the specific dissipation rate, $\omega$ are obtained from the following transport equations:

$$
\begin{aligned}
& \frac{\partial}{\partial t}(\rho k)+\frac{\partial}{\partial x_{i}}\left(\rho k u_{i}\right)=\frac{\partial}{\partial x_{j}}\left(\Gamma_{k} \frac{\partial k}{\partial x_{j}}\right)+G_{k}-Y_{k}+S_{k} \\
& \frac{\partial}{\partial t}(\rho \omega)+\frac{\partial}{\partial x_{i}}\left(\rho \omega u_{i}\right)=\frac{\partial}{\partial x_{j}}\left(\Gamma_{\omega} \frac{\partial \omega}{\partial x j}\right)+G_{\omega}-Y_{\omega}+S_{\omega}
\end{aligned}
$$


$G_{k}$ represents the generation of turbulence kinetic energy due to mean velocity gradients, $G_{k}$ represents the generation of $\omega, \Gamma_{k}$ and $\Gamma_{\omega}$ represent the effective diffusivity of $k$ and $\omega$, respectively, $Y_{k}$ and $Y_{\omega}$ represent the dissipation of $k$ and $\omega$ due to turbulence, $S_{k}$ and $S_{\omega}$ are user-defined source terms.

The effective diffusivities for the $k-\omega$ model are given by:

$$
\Gamma_{k}=\mu+\frac{\mu_{t}}{\sigma_{k}} \quad \text { and } \quad \Gamma_{\omega}=\mu+\frac{\mu_{t}}{\sigma_{\omega}}
$$

Where $\sigma_{k}$ and $\sigma_{\omega}$ are the turbulent Prandlt numbers for $k$ and $\omega$, respectively. The turbulent viscosity $\mu_{t}$ is computed by combining $k$ and $\omega$ as follows:

$$
\mu_{t}=\alpha^{*} \frac{\rho k}{\omega}
$$

The production of turbulence kinetic energy $G_{k}$ may be defined as:

$$
G_{k}=-\rho \overline{u_{i}^{\prime} u_{j}^{\prime}} \frac{\partial u_{j}}{\partial x_{i}}
$$

To evaluate $G_{k}$ in a manner consistent with the boussinesq hypothesis: $G_{k}=\mu_{t} S^{2}$.

The production of $\omega$ is given by:

$$
G_{\omega}=\alpha \frac{\omega}{k} G_{k}
$$

The dissipation of $k$ is giving by:

$$
Y_{k}=\rho \beta^{*} f_{\beta^{*}} k \omega
$$

The dissipation of $\omega$ is giving by:

$$
Y_{\omega}=\rho \beta^{*} f_{\beta} \omega^{2}
$$

$Y_{k}$ and $Y_{\omega}$ represents the dissipations of $k$ and $\omega$, and is defined in a similar manner as in the standard $k-\omega$ model.

Model constants are:

$$
\begin{aligned}
& \sigma_{k, 1}=1.176, \sigma_{\omega, 1}=2.0, \sigma_{k, 2}=1.0, \sigma_{\omega, 2}=1.168 \\
& a_{1}=0.31 \beta_{i, 1}=0.075, \quad \beta_{i, 2}=0.0828, \quad \kappa=0.41
\end{aligned}
$$

\section{5. k- $\omega S S T$ model}

The shear-stress transport (SST) $k-\omega$ model was developed by Menter [MEN 94] to effectively blend the robust and accurate formulation of the $k-\omega$ model in the near-wall region with the freestream independence of the $k-\omega$ model in the far field.

The $k-\omega$ SST model has a similar form to the standard $k-\omega$ model: 
$\frac{\partial}{\partial t}(\rho k)+\frac{\partial}{\partial x_{i}}\left(\rho k u_{i}\right)=\frac{\partial}{\partial x_{j}}\left(\Gamma_{k} \frac{\partial k}{\partial x_{j}}\right)+G_{k}-Y_{k}+S_{k}$

[24]

$$
\frac{\partial}{\partial t}(\rho \omega)+\frac{\partial}{\partial x_{i}}\left(\rho \omega u_{i}\right)=\frac{\partial}{\partial x_{j}}\left(\Gamma_{\omega} \frac{\partial \omega}{\partial x j}\right)+G_{\omega}-Y_{\omega}+D_{\omega}+S_{\omega}
$$

Where $D_{\omega}$ represents the cross-diffusion term.

The turbulent viscosity $\mu_{t}$ is computed as follows:

$$
\mu_{t}=\frac{\rho k}{\omega} \frac{1}{\max \left[\frac{1}{\alpha^{*}}, \frac{S F_{2}}{a_{1} \omega}\right]}
$$

Where: $\sigma_{k}=\frac{1}{\frac{F_{1}}{\sigma_{k, 1}}+\frac{\left(1-F_{1}\right)}{\sigma_{k, 2}}}$ and $\sigma_{\omega}=\frac{1}{\frac{F_{1}}{\sigma_{\omega, 1}}+\frac{\left(1-F_{1}\right)}{\sigma_{\omega, 2}}}$

$F_{1}$ and $F_{2}$ are the blending functions,

The term $G_{k}$ represents the production of turbulence kinetic energy, and is defined as:

$$
G_{k}=\min \left(G_{k}, 10 \rho \beta^{*} k \omega\right)
$$

The term $G_{\omega}$ represents the production of $\omega$ and is given by:

$$
G_{\omega}=\frac{\alpha}{v_{t}} G_{k}
$$

The $k-\omega$ SST model is based on both the standar $k-\omega$ model and the standard $k-\varepsilon$ model. To blend these two models together, the standard $k-\varepsilon$ model has been transformed into equations based on $k$ and $\omega$, which leads to the introduction of a cross-diffusion term $D_{\omega}$ is defined as:

$$
D_{\omega}=2\left(1-F_{1}\right) \rho \sigma_{\omega, 2} \frac{1}{\omega} \frac{\partial k}{\partial x_{j}} \frac{\partial \omega}{\partial x_{j}}
$$

Model constants are:

$$
\begin{gathered}
\sigma_{k, 1}=1.176, \sigma_{\omega, 1}=2.0, \sigma_{k, 2}=1.0, \sigma_{\omega, 2}=1.168 \\
a_{1}=0.31 \beta_{i, 1}=0.075, \beta_{i, 2}=0.0828, \kappa=0.41
\end{gathered}
$$

The $k-\omega$ SST model has the same values as for the standard $k-\omega$ model.

\section{Numerical simulation}

\subsection{Problem statement}

In aerodynamic flow simulation, it is a question of testing the ability of the software to correctly describe the physical phenomena of the flow. So we compare the results of our numerical simulation precisely with experimental results on a generic configuration such as the ONERA M6 wing which 
presents a database for these tests [ELM 17]. This wing was designed by Bernard Monnerie and his colleagues in 1972 within the framework of the AGARD (Advisory Grap for Aerospace Research and Development) cooperation, to serve as an experimental support for three-dimensional flow studies at transonic speeds and high Reynolds numbers (conditions representative of actual thefts of military and civilian aircraft). The database of test results on this wing was documented by Volker Schmitt and François Charpin in the AGARD report published in 1979 [SCH 79].

For this, we carried out an aerodynamic analysis by ANSYS/Fluent software with a Mach number of 0.8395 , an angle of attack of $3.06^{\circ}$ and a Reynolds number until 11.72E6. We validate our aerodynamic simulation results found by ANSYS/Fluent with the database for the ONERA M6 wing consisting on the pressure coefficient $\mathrm{C}_{\mathrm{p}}$, located in different sections $\mathrm{y} / \mathrm{b}=0.2,0.44,0.65,0.95$, and the lift $\left(\mathrm{C}_{1}\right)$ and drag $\left(\mathrm{C}_{\mathrm{d}}\right)$ coefficients [ELM 16] [ELM 17] [ELH 17].

\subsection{Boundary conditions}

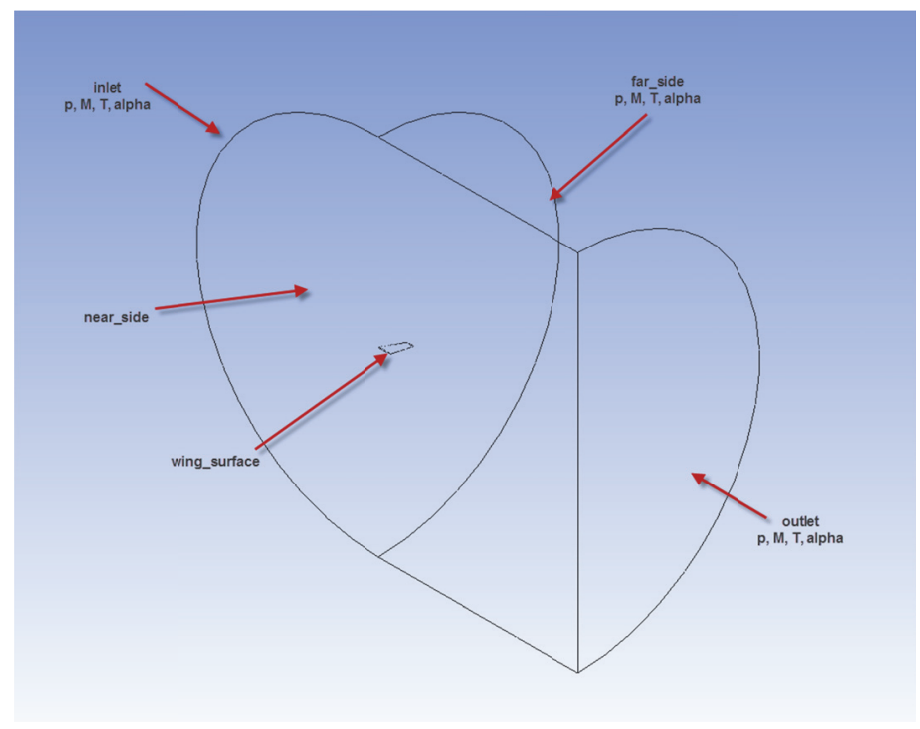

Figure 1. Boundary conditions.

- wing surface: We will be setting this to a wall boundary condition, setting the velocity there to be 0 .

- near side: We will be setting this to symmetry. This basically means the solution is symmetrical with respect to this plane.

- inlet, far side, outlet: We will be setting far-field pressure, Mach number, temperature, and components of the velocity. This allows for the calculation of the speed of sound, and the velocity direction.

A hybrid-unstructured mesh was generated with 375263 cells and 102432 volume nodes points for CFD computations [ELM 17]. It also contains mixtures of tetrahedral, pyramids and prism cells in the boundary layer region. 


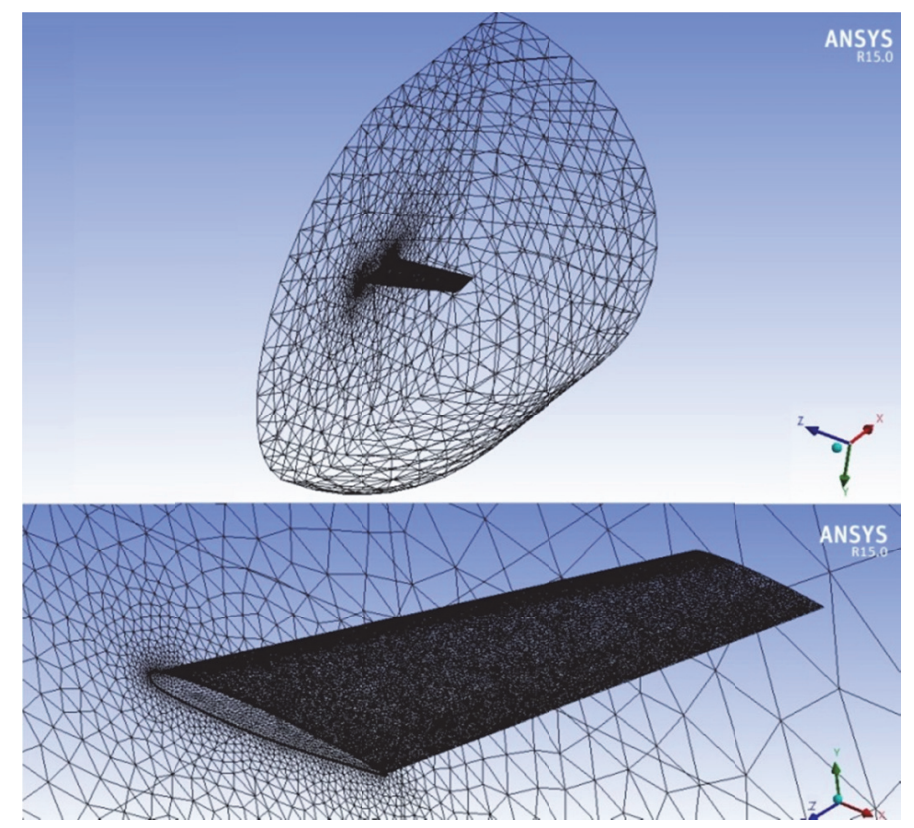

Figure 2. Fluid mesh.

\subsection{Results and Discussions}

In our results, we simulated the pressure coefficient, which is a dimensionless number that describes the relative pressure across a flow field. It is defined by the following formula:

$$
C_{p}=\frac{p-p_{\infty}}{\frac{1}{2} \rho_{\infty} U_{\infty}{ }^{2}}
$$

Where $p_{\infty}$ is the static pressure, $\rho_{\infty}$ is the static density and $U_{\infty}$ is the velocity of flow in infinity. As well as the lift coefficient, reflecting the evolution of the lift force of the wing, and the drag coefficient, representing the resistance of the wing to the air [SCH 79].

Figure 3 illustrates our numerical simulation results of the pressure coefficient using five turbulence models (Spalart-Allmaras, standard k- $\varepsilon, \mathrm{k}-\varepsilon$ RNG, k- $\omega$ Standard and $\mathrm{k}-\omega \mathrm{SST}$ ) compared to experimental results, and Table 1 shows the obtained numerical calculations of the aerodynamic lift and drag coefficients verified by a comparison with the available NASA CFD results. 

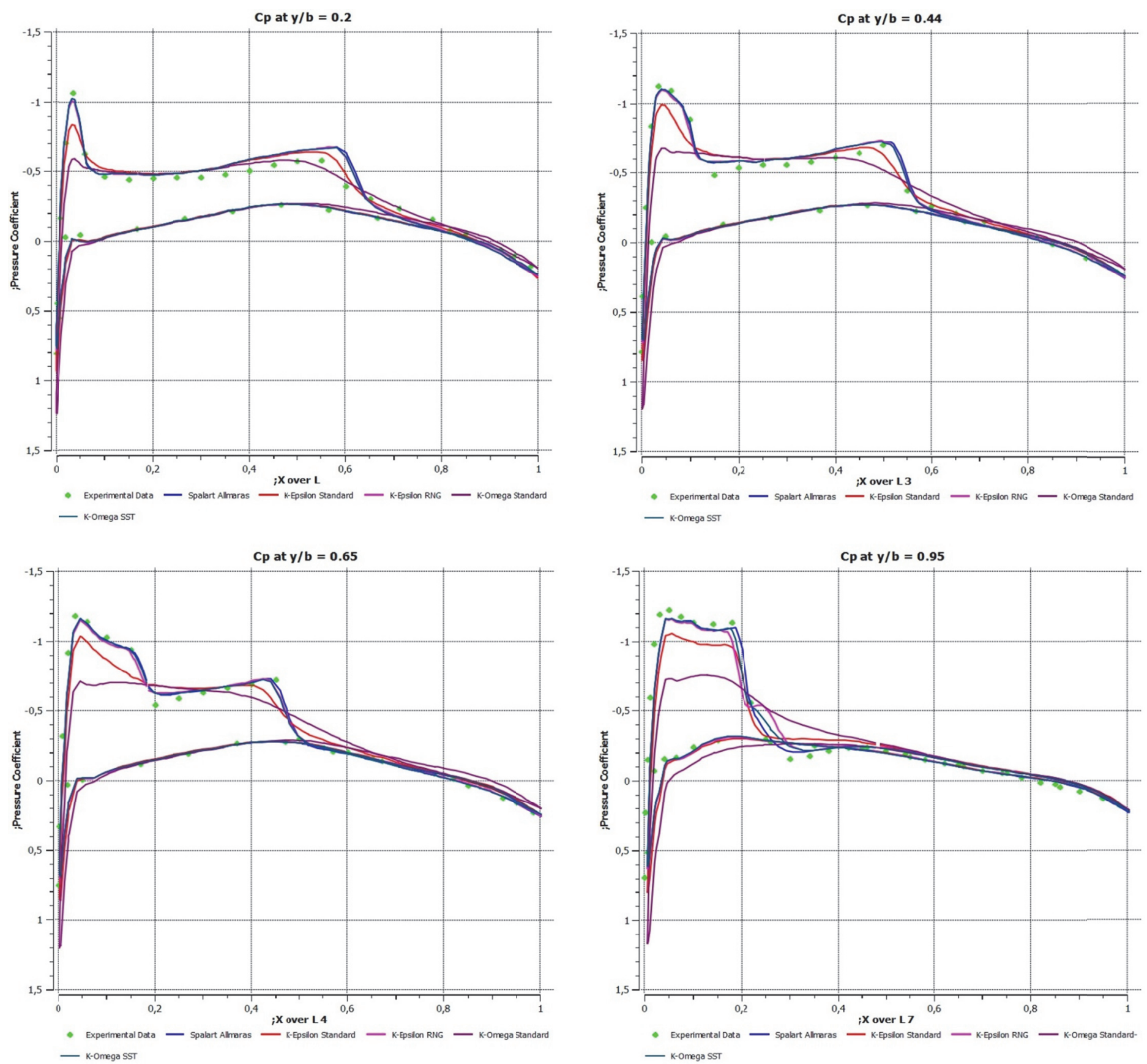

Figure 3. $C_{P}$ Variations for different turbulence models

\begin{tabular}{|c|c|c|c|c|c|c|}
\hline & NASA-CFD & S-A & k- $\boldsymbol{\varepsilon}$ Standard & k- $\boldsymbol{\varepsilon}$ RNG & $\mathbf{k}$ - $\boldsymbol{\omega}$ Standard & k- $\omega$ SST \\
\hline $\begin{array}{c}\text { Lift } \\
\text { coefficient }\end{array}$ & 0.1410 & 0.1310 & 0.1250 & 0.1299 & 0.1102 & 0.1288 \\
\hline $\begin{array}{c}\text { Drag } \\
\text { coefficient }\end{array}$ & 0.0088 & 0.0097 & 0.0145 & 0.0103 & 0.0236 & 0.0096 \\
\hline
\end{tabular}

Table 1. Comparison of lift coefficient and drag coefficient to those from NASA's WIND simulation

Looking at Figure 3, it is easy to see the distribution of the pressure coefficient around the ONERA M6 wing at different places in the wing spanwise locations and compare the results. We can observe first of all that, on the intrados, the numerical simulation with the five models is very close to the experiment, nevertheless, on the extrados, there is a difference using each model, it can be seen that simulations using standard models such as $\mathrm{k}-\varepsilon$ standard and $\mathrm{k}-\omega$ standard give non-regular results 
between 0 and 0.2 as well as between 0.4 and 0.6. However, the three other models (Spalart-Allmaras, $\mathrm{k}-\varepsilon \mathrm{RNG}$ and $\mathrm{k}-\omega \mathrm{SST}$ ) are confused with the experimental results at any section point.

On the other hand, the comparison of the aerodynamic lift and drag coefficients, in Table 1, shows that the results of the numerical simulation data obtained using standard turbulence models for $\mathrm{k}-\varepsilon$ and $\mathrm{k}-\omega$ are far from the results of the CFD applied by NASA, nevertheless, the simulations by the other

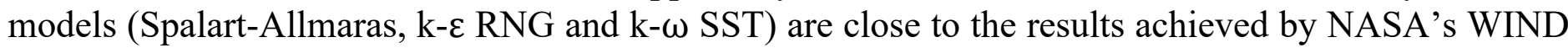
simulation.

\section{Conclusion}

Our study focused on several aspects; first it was first necessary to perform numerical simulations of the flow around the ONERA M6 wing using ANSYS/FLUENT software, to validate the numerical simulation around this wing. Secondly, this aerodynamic flow was modeled by five different models

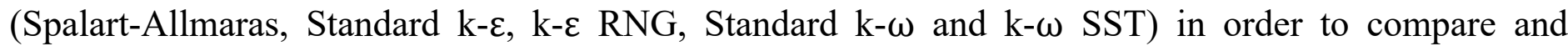
validate the most efficient model in this simulation. The main conclusion that can be drawn from this stage of the study for the pressure coefficient results is that the five turbulence models have the same general behavior for with some differences in the pressure values on the upper surface. Also for the values of the aerodynamic lift and drag coefficients, we conclude that the Spalart-Allmaras, k- $\varepsilon$ RNG and k- $\omega$ SST models give closer results to the NASA CFD results. Our study thus tends to show that the Spalart-Allmaras, $k-\varepsilon$ RNG and k- $\omega$ SST models are the most efficient models to model the turbulence of the transonic flow around an ONERA M6 wing.

\section{Bibliography}

[ELH 17] El HAMI A., RADI B., Fluid-Structure Interactions and Uncertainties: Ansys and Fluent Tools, Wiley-iSTE, 2017.

[ELM 17] El MAANI R., Étude basée sur l'optimisation fiabiliste en aérodynamique, Thèse de doctorat, INSA de Rouen, 2016.

[ELM 17] El MAANi R., Elmakhloufi A., RADi B. AND El HAMI A., RBDO analysis of the aircraft wing based aerodynamic behavior, Structural Engineering and Mechanics, 61(4), 441-451, 2017.

[ELM 17] El MAANI R., RADI B. AND El HAMI A., Vibratory Reliability Analysis of an Aircraft's Wing via FluidStructure Interactions, Aerospace, MDPI, 4(3), 40, 2017.

[LAU 72] Launder B. E. AND Spalding D. B., Lectures in Mathematical Models of Turbulence, Academic Press, London, England, 1972.

[MEN 94] MENTER F. R., Two-Equation Eddy-Viscosity Turbulence Models for Engineering Applications, AIAA Journal, 32(8), 1598-1605, 1994.

[SCH 79] SCHMITT V. AND CHARPIN F., Pressure distributions on the onera m6 wing at transonic mach numbers, Agardar-138-experimental data base for computer program assessment, 1979.

[SPA 93] SPALART P. R., AND AlLMARAS S.R., A one-equation turbulence model for aerodynamic flows, AIAA Paper 1993-0439.

[WIL 94] WILCOX D. C., Turbulence Modeling for CFD, DCW Industries, 1994.

[WIL 98] WILCOX D. C., Turbulence Modeling for CFD, DCW Industries, Inc. La Canada, California. 1998. 